\title{
RR Lyrae spectroscopic observations over a whole Blazhko period: frequency analysis and implications for theoretical models ${ }^{\star}$
}

\author{
M. Chadid ${ }^{1,2,3}$ and E. Chapellier ${ }^{3}$ \\ 1 LUAN, UMR 6525, University of Nice Sophia-Antipolis, Parc Valrose, 06108 Nice Cedex 02, France \\ 2 ConcordiAstro, Concordia Station, Dome C, TAAF, Antarctica \\ 3 Observatoire de la Côte d'Azur, UMR 6203, BP 4229, 06304 Nice Cedex 04, France \\ e-mail: chadid@unice.fr
}

Received 11 August 2005 / Accepted 26 March 2006

ABSTRACT

\begin{abstract}
Context. We perform a new spectroscopy of RR Lyrae to gain an understanding of the Blazhko effect. The Blazhko effect is a periodic amplitude and/or phase modulation shown by some RR Lyrae stars.

Aims. New high precision spectroscopic observations of RR Lyrae, the brightest Blazhko star, were obtained continuously over an entire Blazhko period (41 days), from June to August 2000. This data set allows for a detailed analysis.

Methods. We used Fourier techniques to analyze the metallic absorption line-profile variations over the pulsation and the Blazhko cycle.

Results. This data set shows a triplet frequency structure with a large asymmetry in the modulation amplitudes. The right sidepeak amplitudes are higher than those on the left, and there is a linear decrease of the amplitudes of both right and left modulation components. The new data reveal that there is a very narrow interval in the pulsation cycle, $20 \%$ of the pulsation period, in which the modulation shows intense variations. The interval occurs during the phase of the main shock passage across the atmosphere of RR Lyrae. We detected no evidence whatsoever of a quintuplet structure within a median $1 \sigma$ radial velocity uncertainty of $250 \mathrm{~m} / \mathrm{s}$. Our spectroscopic results make it more difficult to explain the Blazhko phenomenon with the current resonance models, which are the only theoretical models available that explain the Blazhko phenomenon, and give new insight for future Blazhko theoretical investigations.
\end{abstract}

Key words. line: profiles - stars: variables: RR Lyrae - stars: oscillations - techniques: spectroscopic

\section{Introduction}

RR Lyrae stars play a major astrophysical role as standard candles for distance determination and as witnesses of the evolution of the universe at a young age. These variables occupy a special place in the family of pulsating variable stars. They have been known for more than a century because of their large amplitude and cycle lengths. Until recently, these stars were considered to be prototypes of pure radial pulsators, with periods ranging from a few hours up to a whole day.

Although these stars have been well studied, major questions concerning RR Lyrae stars still remain. Chief among these is the origin of the so-called Blazhko effect (Blazhko 1907), a periodic modulation of both the amplitude and the phase of the main pulsation on timescales typically varying from dozens of days to hundreds of days. This phenomenon is exhibited by some $25-30 \%$ of the RRab and $5 \%$ of the RRc population of the Galactic Bulge (Moskalik \& Poretti 2003). In the LMC, its occurrence among the RRab stars is only half as large (Alcock et al. 2003).

The Blazhko effect does not yet have a generally accepted explanation. The investigation of the physical origin of the Blazhko effect is extremely important for understanding the nature of stellar pulsation. Several explanations have been put forward, but there is still no consensus on the mechanism

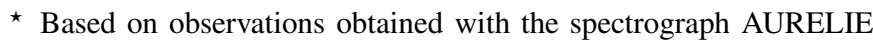
attached to the $1.52 \mathrm{~m}$ telescope of the Haute-Provence Observatory, France. underlying the Blazhko effect (Kovàcs 1995, 2001). Both groups of competing Blazhko models that exist today involve nonradial pulsational modes. We see modulations that, by their nature, beat frequency components. Unfortunately, none of the published spectroscopic papers brought us closer to the nature of the modulation. The first observational detection that the Blazhko phenomenon is caused by nonradial mode excitation comes from analysis of RR Lyrae high precision spectroscopic observations (Chadid et al. 1999), showing a triplet frequency structure with a separation equal to the Blazhko frequency around the pulsation frequency and the left sidepeak amplitude higher than that on the right. Chadid et al. claimed a quintuplet structure as well. Later et al. (2000) and Smith et al. (2003), basing their position on CCD photometric observations, confirmed the triplet frequency structure in RR Lyrae, but with a higher right sidepeak. They did not detect any quintuplet structure.

Nevertheless, it has still been hard to identify exactly which nonradial pulsation modes are excited in RR Lyrae, based primarily on observations poorly spread over both the pulsation and the Blazhko period, in combination with the time spacing of the observations, which introduces alias frequencies and can bias the observed amplitudes of the detected frequencies.

In this first part of a series of two papers on RR Lyrae, we present high precision spectroscopic observations spread continuously over a whole Blazhko period (41 days). We perform a detailed frequency analysis of the profile variations of Fe II $\lambda \lambda 4923.921$, comparing them with prior results from spectroscopic and CCD photometric observations. In the second 
Table 1. Journal of AURELIE spectroscopic observations of RR Lyrae at the $1.52 \mathrm{~m}$ Telescope.

\begin{tabular}{|c|c|c|c|}
\hline Date & $\begin{array}{c}\text { HJD } \\
2400000+\end{array}$ & $\begin{array}{c}\text { Number of } \\
\text { spectra }\end{array}$ & $\begin{array}{l}\text { Max/Min } \\
\left(\mathrm{km} \mathrm{s}^{-1}\right)\end{array}$ \\
\hline 2000 Jun. 27 & 51723 & 51 & $-45.00 /-105.20$ \\
\hline 2000 Jun. 28 & 51724 & 25 & $-47.80 /-66.33$ \\
\hline 2000 Jun. 29 & 51725 & 53 & $-52.48 /-89.30$ \\
\hline 2000 Jun. 30 & 51726 & 61 & $-72.61 /-108.32$ \\
\hline 2000 Jul. 01 & 51727 & 41 & $-45.66 /-107.32$ \\
\hline 2000 Jul. 04 & 51730 & 67 & $-61.65 /-107.80$ \\
\hline 2000 Jul. 05 & 51731 & 65 & $-45.00 /-108.80$ \\
\hline 2000 Jul. 06 & 51732 & 60 & $-45.35 /-99.68$ \\
\hline 2000 Jul. 07 & 51733 & 61 & $-48.84 /-77.57$ \\
\hline 2000 Jul. 08 & 51734 & 58 & $-56.60 /-102.71$ \\
\hline 2000 Jul. 09 & 51735 & 69 & $-45.01 /-106.63$ \\
\hline 2000 Jul. 10 & 51736 & 34 & $-46.33 /-107.70$ \\
\hline 2000 Jul. 12 & 51738 & 62 & $-52.68 /-96.289$ \\
\hline 2000 Jul. 13 & 51739 & 25 & $-89.21 /-106.94$ \\
\hline 2000 Jul. 14 & 51740 & 46 & $-48.49 /-106.51$ \\
\hline 2000 Jul. 15 & 51741 & 45 & $-51.73 /-64.85$ \\
\hline 2000 Jul. 16 & 51742 & 85 & $-55.22 /-89.33$ \\
\hline 2000 Jul. 17 & 51743 & 75 & $-67.07 /-104.53$ \\
\hline 2000 Jul. 18 & 51744 & 90 & $-49.55 /-104.65$ \\
\hline 2000 Jul. 20 & 51746 & 100 & $-54.90 /-84.541$ \\
\hline 2000 Jul. 21 & 51747 & 58 & $-71.23 /-97.22$ \\
\hline 2000 Jul. 22 & 51748 & 87 & $-48.30 /-101.05$ \\
\hline 2000 Jul. 24 & 51750 & 73 & $-50.40 /-76.17$ \\
\hline 2000 Jul. 26 & 51752 & 100 & $-45.60 /-98.10$ \\
\hline 2000 Jul. 28 & 51754 & 63 & $-48.01 /-78.27$ \\
\hline 2000 Jul. 29 & 51755 & 51 & $-55.75 /-95.18$ \\
\hline 2000 Jul. 30 & 51756 & 98 & $-44.30 /-100.01$ \\
\hline 2000 Jul. 31 & 51757 & 99 & $-43.20 /-100.20$ \\
\hline 2000 Aug. 01 & 51758 & 44 & $-42.01 /-61.34$ \\
\hline 2000 Aug. 02 & 51759 & 71 & $-51.44 /-94.37$ \\
\hline 2000 Aug. 04 & 51761 & 97 & $-41.20 /-101.61$ \\
\hline 2000 Aug. 05 & 51762 & 77 & $-40.25 /-90.75$ \\
\hline 2000 Aug. 06 & 51763 & 88 & $-44.84 /-88.56$ \\
\hline
\end{tabular}

paper, we will show the connection between the temporal hydrodynamic phenomena in the atmosphere of RR Lyrae and the occurence of modulations.

\section{Observations and data processing}

\subsection{High resolution spectroscopic observations and data reduction}

The spectroscopic observations used in this study were obtained at the Observatoire de Haute-Provence from June to August 2000, using the AURELIE spectrograph attached to the $1.52 \mathrm{~m}$ telescope. The detector used was CCD-EEV42-20, with $2048 \times 1024$ elements of size $13.5 \mu \mathrm{m}^{2}$. The observations were obtained over a timespan of forty-one days (the RR Lyrae Blazhko period), with observations obtained on thirty-three nights (see Table 1). The spectral domain covers the range from $484 \mathrm{~nm}$ to $494 \mathrm{~nm}$ with resolving power $R \simeq 60000$. A signalto-noise ratio $(\mathrm{S} / \mathrm{N})$ of around 90 was obtained for an exposure time between $3-5 \mathrm{mn}$, which corresponds to a phase resolution around $0.5 \%$ of the pulsation period of $13 \mathrm{~h} 36 \mathrm{mn}$. In this study, we have used the profile of the single ionized metallic absorption line Fe II $\lambda \lambda$ 4923.921. The AURELIE optimal data reduction of the CCD images was carried out using the Munich Image Data Analysis System (MIDAS). We treated the 2179 spectra in the same way. A detailed description of the data reduction can be found in Chadid \& Gillet $(1996,1997)$. During this run, the mechanical thermal and optical characteristics of the spectrograph did not change. At the beginning of each night, the positioning of the echelle orders on the CCD was calibrated. The wavelength calibration was done using a thorium lamp. A thorium arc was taken at the beginning and the end of each night. To confirm the correct operation of the AURELIE instrument and more particularly the estimated error of the radial velocity measurements, we obtained thirty-three observations of the radial velocity of the standard star HD 168009 (G2V spectral type and $-64.4 \mathrm{~km} \mathrm{~s}^{-1}$ radial velocity, according to Perryman et al. 1997) at the beginning of each night simultaneously. Calibration data was performed in the same manner as the RR Lyrae spectra. The S/N ratio in the HD 168009 spectra is typically around 100.

\subsection{Radial velocity measurements}

The radial velocity measurements were obtained with both simple Gaussian fit and first line moment methods. As the differences between both methods occur only at certain phases in the pulsation cycle, when the line profiles were extremely broad, and moreover when line doubling occurs due to a shock wave (Chadid \& Gillet 1996a,b), we choose the first line moment method for all analyses in this paper.

The radial velocity measurements of the standard star HD 168009 were obtained in the same manner and showed uncertainties in the range $200-250 \mathrm{~m} / \mathrm{s}$.

\section{Frequency analysis results}

\subsection{Fourier analysis}

As can be seen in Fig. 1, the data sampling provides a homogeneous coverage of all Blazhko phases. The variation in both heights of maxima and minima of the radial velocity curves indicated a modulation period of about 41 days.

The frequency analysis is carried out using the standard discrete Fourier transform for unequally spaced data (Deeming 1975) with prewhitening (i.e., successive substraction of the signal component having the largest amplitude and its harmonics) performed on the variations of the heliocentric radial velocity of the metallic absorption line profile Fe II $\lambda$ 4923.921. We used the Period04 software from Lenz \& Breger (2003) that allows prewhitening of selected frequencies. This is done by a leastsquares technique. The Fourier transform analysis consists of fitting the heliocentric radial velocity measurements by means of the series

$I(t)=A_{0}+\sum_{i=1, n} A_{i} \sin \left[2 \pi f_{i}\left(t-T_{0}\right)+\Phi_{i}\right]$

Where $T_{0}$ is the initial epoch value of the data set, HJD $T_{0}=$ 2451723.3769.

The Fourier transform analysis on the heliocentric radial velocity data showed a pulsation frequency $f_{0}=1.7637 \mathrm{c} / \mathrm{d}$ $\left(P_{P}=0.5669 \mathrm{~d}\right)$ as the dominant frequency with its first fourteen harmonics, all amplitudes above the noise level (see Table 2, Fig. 2). The Fourier spectra prewhitened with the pulsation frequency and its harmonics are shown in Fig. 2 (the second panel from the top). Prominent peaks are seen at frequencies of 1.7914 , $3.5538,5.3157,7.0771,8.8412,10.6051,12.3668,14.0768$, and $15.9002 \mathrm{c} /$ day. These frequencies beat against $f_{0}$ with beat periods of 36.10, 37.88, 40.65, 44.84, 44.05, 43.67, 47.85, 36.76, and 37.17 days, consistent with a 41 day Blazhko period.

The third panel from the top of Fig. 2 shows the results of the Fourier transform analysis after a second prewhitening 


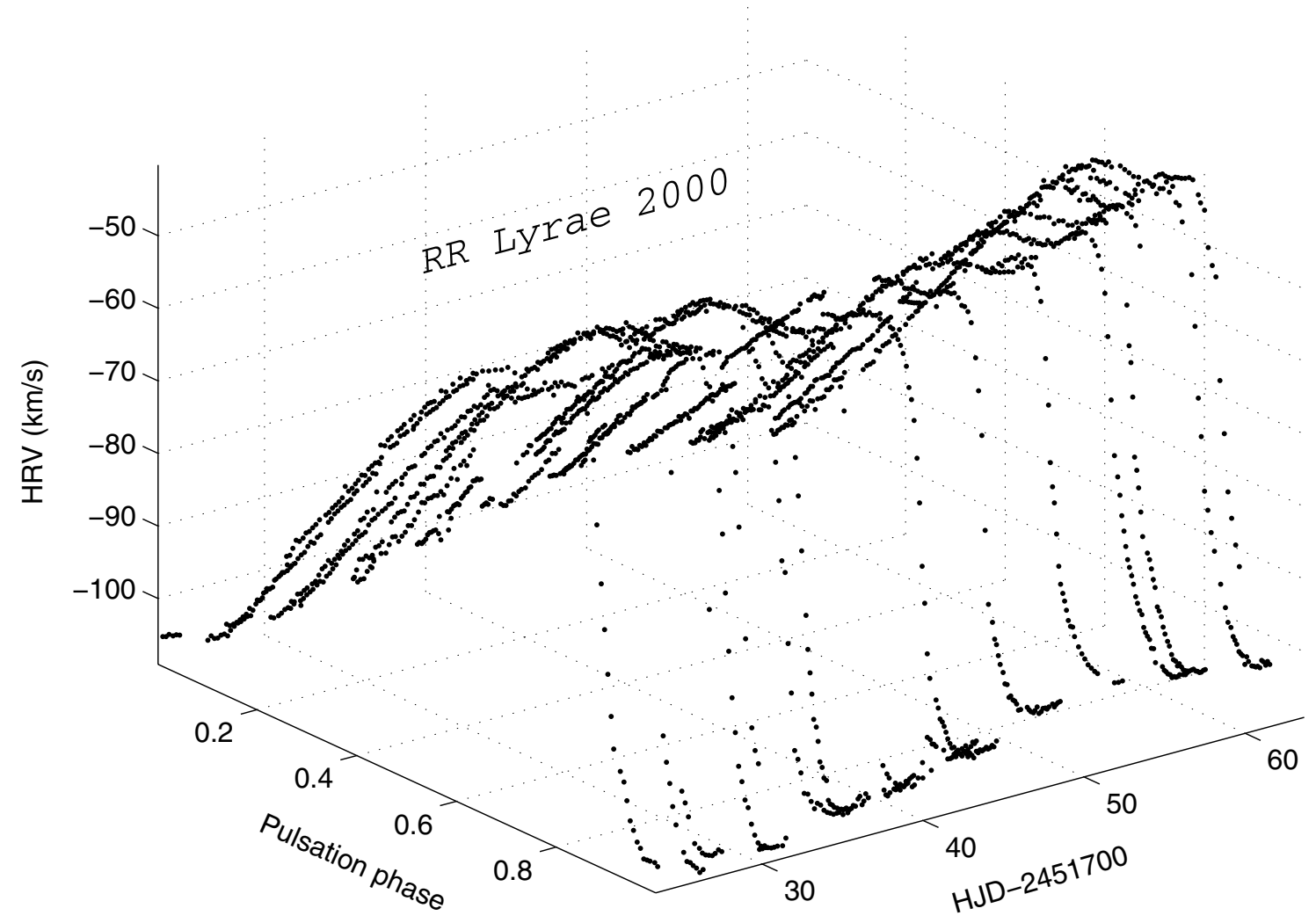

Fig. 1. Two-dimensional radial velocity curve (in $\mathrm{km} \mathrm{s}^{-1}$ ) calculated from the AURELIE spectra and folded with the pulsation period (0.5669 d) over a whole Blazhko cycle. The variations in the radial velocity curve due to the Blazhko effect are clearly visible. The pulsation phase has been calculated from the ephemeris given by Chadid \& Gillet (1997).

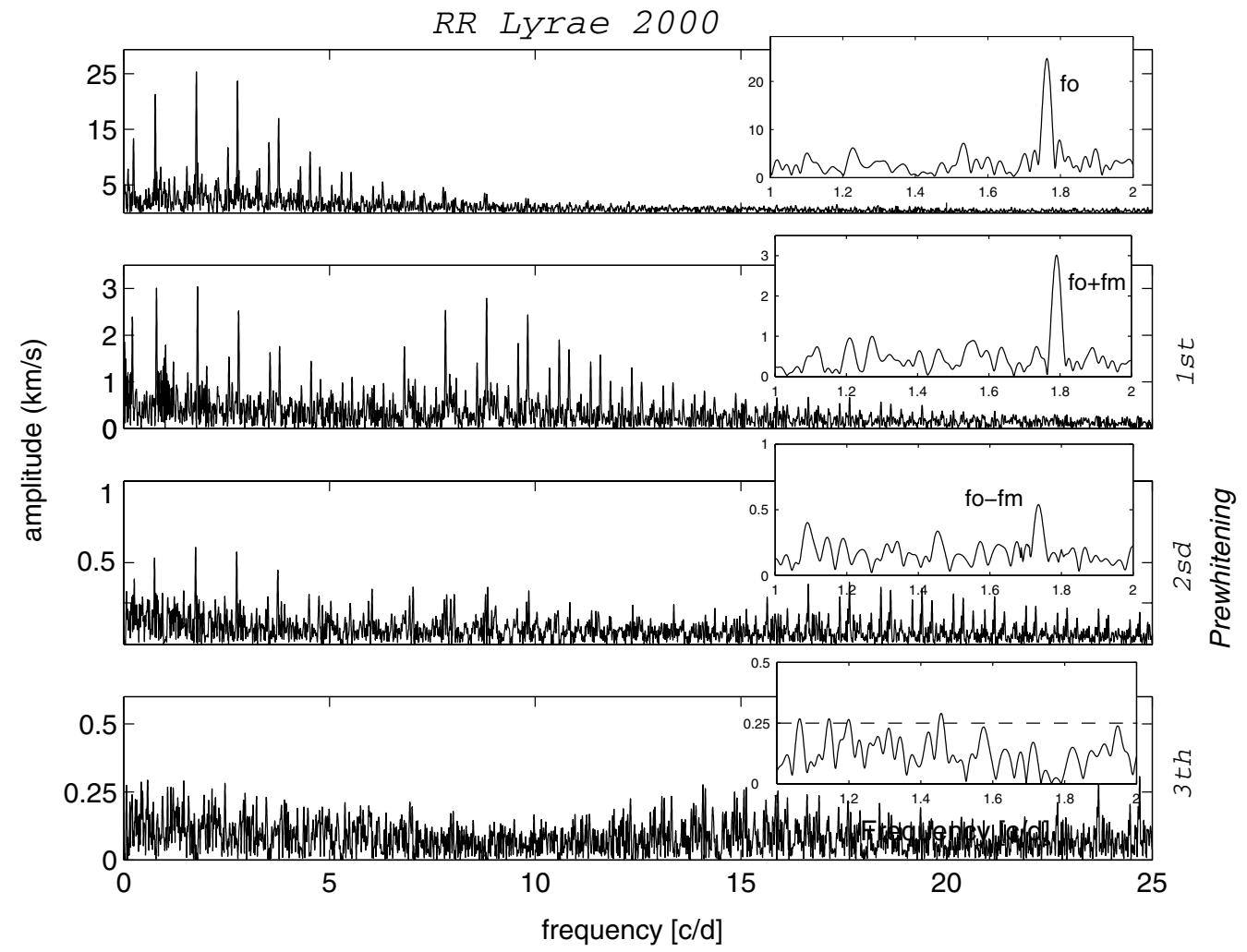

Fig. 2. Fourier spectra (from all 2179 spectra) on the heliocentric radial velocity. The upper panel shows the amplitude spectrum, the other three panels are the residual spectrum after prewhitening with the main frequency $f_{0}$ and its harmonics, the $k f_{0}+f_{m}$ frequency and the $k f_{0}-f_{m}$ frequency respectively. The insert panels show the enlargements between the frequencies $1.00 \mathrm{c} / \mathrm{d}$ and $2.00 \mathrm{c} / \mathrm{d}$. The dashed curve shows the noise level that corresponds to the level of the weakest amplitude in the data. 
Table 2. Observed frequencies (in c/d) of spectral line variations in RR Lyrae and their observed amplitudes and phases resulting from a Fourier analysis.

\begin{tabular}{lccl}
\hline \hline $\begin{array}{l}\text { Frequency } \\
(\mathrm{c} / \mathrm{d})\end{array}$ & $\begin{array}{c}\text { Amplitude } \\
\left(\mathrm{km} \mathrm{s}^{-1}\right)\end{array}$ & $\begin{array}{c}\text { Phase } \\
\text { (cycles) }\end{array}$ & ID \\
\hline 1.7637 & 24.3664 & 0.3161 & $f_{0}$ \\
3.5274 & 9.2844 & 0.0682 & $2 f_{0}$ \\
5.2912 & 6.0379 & 0.8317 & $3 f_{0}$ \\
7.0550 & 4.1191 & 0.6159 & $4 f_{0}$ \\
1.7914 & 3.1963 & 0.0819 & $f_{0}+f_{m}$ \\
8.8184 & 2.6986 & 0.4202 & $5 f_{0}$ \\
10.5810 & 1.7527 & 0.2523 & $6 f_{0}$ \\
3.5538 & 1.2967 & 0.7975 & $2 f_{0}+f_{m}$ \\
12.3447 & 1.1494 & 0.0412 & $7 f_{0}$ \\
5.3157 & 1.0679 & 0.5638 & $3 f_{0}+f_{m}$ \\
7.0771 & 0.9816 & 0.4321 & $4 f_{0}+f_{m}$ \\
3.4985 & 0.8928 & 0.9685 & $2 f_{0}-f_{m}$ \\
14.1131 & 0.8660 & 0.7493 & $8 f_{0}$ \\
5.2671 & 0.7624 & 0.5871 & $3 f_{0}-f_{m}$ \\
8.8412 & 0.7603 & 0.1868 & $5 f_{0}+f_{m}$ \\
15.8757 & 0.7564 & 0.5592 & $9 f_{0}$ \\
7.0336 & 0.7330 & 0.3685 & $4 f_{0}-f_{m}$ \\
17.6390 & 0.6151 & 0.3554 & $10 f_{0}$ \\
1.7343 & 0.6071 & 0.1582 & $f_{0}-f_{m}$ \\
10.6051 & 0.5853 & 0.9175 & $6 f_{0}+f_{m}$ \\
8.7998 & 0.5750 & 0.1104 & $5 f_{0}-f_{m}$ \\
19.4036 & 0.4835 & 0.1311 & $11 f_{0}$ \\
12.3668 & 0.4624 & 0.7271 & $7 f_{0}+f_{m}$ \\
21.1677 & 0.4263 & 0.9324 & $12 f_{0}$ \\
10.5574 & 0.3953 & 0.0461 & $6 f_{0}-f_{m}$ \\
22.9309 & 0.3312 & 0.7353 & $13 f_{0}$ \\
12.3170 & 0.3107 & 0.9203 & $7 f_{0}-f_{m}$ \\
14.1368 & 0.3106 & 0.8359 & $8 f_{0}+f_{m}$ \\
24.6945 & 0.3043 & 0.5291 & $14 f_{0}$ \\
15.9002 & 0.2641 & 0.2224 & $9 f_{0}+f_{m}$ \\
26.4587 & 0.2466 & 0.3131 & $15 f_{0}$ \\
\hline & & & \\
\hline & & & \\
& & &
\end{tabular}

of the modulation frequencies. We point out that peaks at frequencies of $1.7343,3.4985,5.2671,7.0336,8.7998,10.5574$, and $12.3170 \mathrm{c} /$ day beat against $f_{0}$ with periods of $34.01,34.60$, $41.67,47.17,53.48,40.32$, and 34.60 days, with an average of around 40.84 days, which is consistent with the Blazhko period as well. A third prewhitening of the all modulation frequencies gave a flat spectrum, showing that there are no other components present above the noise level (see the bottom panel in Fig. 2). We could have extended the Fourier analysis below the noise level by imposing the frequencies that correspond to each harmonic, but we preferred to limit our analysis to the peaks that are well above the noise level.

In summary, the Fourier spectrum analysis shows thirtyone frequencies (see Table 2), the dominant radial frequency $f_{0}=1.7637 \mathrm{c} / \mathrm{d}$ and its first fourteen harmonics and the triplet structure of the amplitude modulation up to about the 7 th harmonic component (see Fig. 3).

We also re-performed the analysis with fixed values of the mean frequency and its harmonics and the right modulation frequencies. The frequencies $f_{p}$ and $f_{b}$ were calculated from the pulsation period and Blazhko period taken from the ephemerides of Chadid et al. (1997). Note that only the frequency values were considered as input values (known parameters), and not their amplitudes or their phases. Prewhitening with the main frequency and its harmonics and the right modulation frequencies revealed, as expected, the existence of the left modulation frequencies. Table 3 lists the results of a multifrequency fit according to this method. The results are similar to those of the previous method

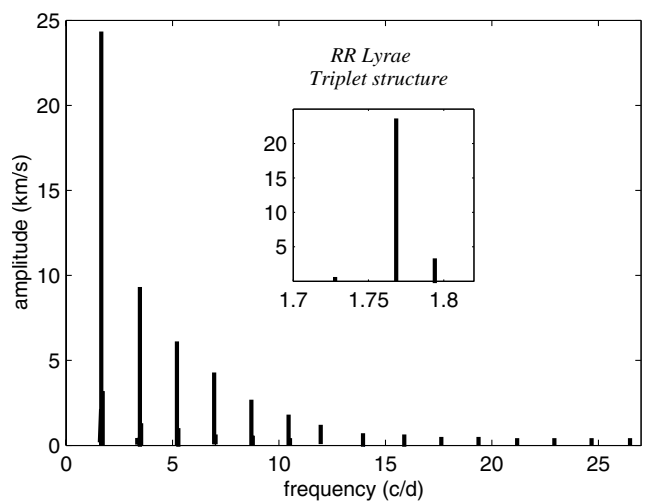

Fig. 3. The frequency spectrum of RR Lyrae. The insert panel shows the triplet structure at the main frequency $f_{0}$.

Table 3. Like Table 2, with $f_{p}$ and $f_{p}+f_{b}$ frequencies taken from Chadid et al. (1999).

\begin{tabular}{lccl}
\hline \hline $\begin{array}{l}\text { Frequency } \\
\text { (c/d) }\end{array}$ & $\begin{array}{c}\text { Amplitude } \\
\left(\mathrm{km} \mathrm{s}^{-1}\right)\end{array}$ & $\begin{array}{c}\text { Phase } \\
\text { (cycles) }\end{array}$ & ID \\
\hline 1.7642 & 24.59974 & 0.30414 & $f_{p}$ \\
3.5283 & 8.95331 & 0.04411 & $2 f_{p}$ \\
5.2925 & 5.98857 & 0.80582 & $3 f_{p}$ \\
7.0567 & 4.07245 & 0.59028 & $4 f_{p}$ \\
1.7887 & 3.41380 & 0.15834 & $f_{p}+f_{b}$ \\
8.8208 & 2.74662 & 0.38618 & $5 f_{p}$ \\
10.585 & 1.73655 & 0.17883 & $6 f_{p}$ \\
3.5528 & 1.28887 & 0.84408 & $2 f_{p}+f_{b}$ \\
12.3492 & 1.22585 & 0.95239 & $7 f_{p}$ \\
5.3170 & 0.97589 & 0.57735 & $3 f_{p}+f_{b}$ \\
7.0812 & 0.81483 & 0.3885 & $4 f_{p}+f_{b}$ \\
3.5170 & 0.57084 & 0.33854 & $2 f_{p}-f_{b}$ \\
14.1134 & 0.92667 & 0.73025 & $8 f_{p}$ \\
5.2678 & 0.6860 & 0.48906 & $3 f_{p}-f_{b}$ \\
8.8454 & 0.56117 & 0.16056 & $5 f_{p}+f_{b}$ \\
15.8775 & 0.77373 & 0.52221 & $9 f_{p}$ \\
7.0281 & 0.46506 & 0.42436 & $4 f_{p}-f_{b}$ \\
17.6417 & 0.56209 & 0.29628 & $10 f_{p}$ \\
1.7396 & 0.64785 & 0.01248 & $f_{p}-f_{b}$ \\
10.6095 & 0.37813 & 0.91225 & $6 f_{p}+f_{b}$ \\
8.7912 & 0.32202 & 0.21899 & $5 f_{p}-f_{b}$ \\
19.4059 & 0.47079 & 0.08329 & $11 f_{p}$ \\
12.3737 & 0.23781 & 0.66872 & $7 f_{p}+f_{b}$ \\
21.1700 & 0.39798 & 0.88133 & $12 f_{p}$ \\
10.5472 & 0.21005 & 0.23726 & $6 f_{p}-f_{b}$ \\
22.9342 & 0.26987 & 0.65634 & $13 f_{p}$ \\
12.3092 & 0.13197 & 0.03854 & $7 f_{p}-f_{b}$ \\
14.1379 & 0.13494 & 0.42748 & $8 f_{p}+f_{b}$ \\
24.6984 & 0.26278 & 0.43214 & $14 f_{p}$ \\
15.9020 & 0.15717 & 0.19493 & $9 f_{p}+f_{b}$ \\
26.4625 & 0.19525 & 0.22336 & $15 f_{p}$ \\
\hline & & & \\
& & & \\
& & &
\end{tabular}

(Table 2). However, this prescribed frequency method gives an rms residual value of $0.90 \mathrm{~km} \mathrm{~s}^{-1}$ higher than the rms residual value of the previous procedure $\left(0.68 \mathrm{~km} \mathrm{~s}^{-1}\right)$. Therefore, for the present paper we consider, only results given by the previous method (Table 2).

\subsection{Modulation frequencies and amplitudes}

Table 2 summarizes the amplitudes of pulsation and modulation frequencies. As shown in Sect. 3.1, the side lobe frequencies of the triplet are almost symmetrically placed around the radial mode frequency and its harmonics. We computed the degree of 


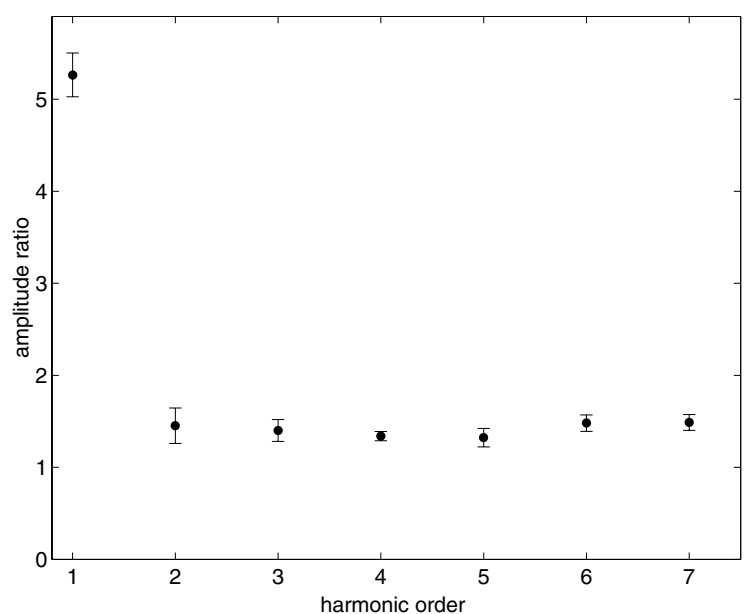

Fig. 4. Side lobe amplitude ratios $\left(A_{k f_{0}+f_{m}} / A_{k f_{0}-f_{m}}\right)$ as a function of harmonic order.

deviation from the equidistant spacing $\left(\delta f=f_{+}+f_{-}-2 f_{0}\right)$ of the triplet structure, and we found $-0.0017 \pm 0.023 \mathrm{c} / \mathrm{d}$.

On the other hand, it can be clearly seen from Figs. 3 and 4 that the modulation amplitude of the higher frequency side of the triplet is larger than its lower frequency counterpart (see also Table 4). The mean value of the degree of asymmetry in the modulation amplitudes $\left(Q=\left(A_{+}-A_{-}\right) /\left(A_{+}+A_{-}\right)\right.$, note: $-1.0 /+1.0$ refers to complete asymmetry, 0 to a symmetric triplet) averaged over the first 7 modulation components is $0.224 \pm 0.010$. This indicates a higher than average asymmetry to the right. The asymmetry in the modulation amplitudes is a significant problem for currently available models to explain the Blazhko phenomenon (Shibahashi 2000; Nowakowski \& Dziembowski 2001; Nowakowski 2002). This fact will be discussed in Sect. 4.

\subsection{Comparison with prior results}

Figure 5 shows exponentially decreasing amplitudes of the harmonics of the main pulsation frequency up to the 14th harmonic component. This is due to nonlinear effects of the radial mode. While the amplitudes of the main pulsation show an exponential decrease, the amplitude decrease of the modulation components is linear up to the 8th harmonic component of the right side lobe and the 6th harmonic component of the left lobe (see Fig. 5, the insert panels). Table 4 summarizes the amplitude ratio values and their corresponding uncertainties. For comparison with the most recent RR Lyrae photometry studies, we computed the amplitude ratios of the harmonic components of the radial pulsation and those of the modulation components from $B$ and $V$ photometric data (Smith et al. 2003, Tables 3 and 4), which are plotted in Fig. 6. We deduced the same exponential phenomenon demonstrating a nonlinear character of the main pulsation. Unfortunately, because of the large dispersion, it is not possible to make a satisfactory conclusion regarding the behaviour of the photometric modulation amplitudes as a function of harmonic order (see Fig. 6). Similar results have been shown by Jurcsik et al. (2004) from photometric data of the Blazhko star RR Gem.

On the other hand, from the $B$ and $V$ photometric analysis (Smith et al. 2003), we computed the degree of asymmetry in the modulation amplitudes and we found an average value of $Q=0.4089$ in the $B$-band and $Q=0.6005$ in the $V$-band. This means that both spectroscopic and photometric results show a large asymmetry in the modulation amplitudes. From MACHO data, a large number of Blazhko variables exhibit an asymmetry of around +0.3 , close to our RR Lyrae spectroscopic value $(Q=0.224)$.

Among the Blazhko variables with triplet structure in the MACHO database, there is a very large number for which the modulation amplitude of the higher frequency side of the triplet is larger than its lower frequency counterpart (Alcock et al. 2003). Our spectroscopic results place RR Lyrae in the same category. However, these results are inconsistent with Chadid et al.'s spectroscopic results (1999), which showed the left sidepeak amplitude higher than that on the right.

\subsection{Irregular behaviour of the Blazhko modulation}

Figures 7, 8, and 9 (lower panel) show the residual radial velocity curves after prewhitening with, respectively, the main pulsation and the side lobes of each triplet, in an attempt to remove the Blazhko modulation from the data set. The curves have been phased according to the HJD $T_{0}=2451723.3769$ initial epoch value of the data set and using $\left(f_{0}+f_{m}\right)^{-1},\left(f_{0}-f_{m}\right)^{-1}$, and $f_{0}^{-1}$ respectively. The most striking character of the residual curves is that the residual plot of all modulation components (Fig. 9 bottom) shows intense variations that appear only in a narrow phase interval of the pulsation cycle, $20 \%$ of the pulsation period. The interval occurs during the descending branch of the radial velocity curve (see Fig. 9). This phenomenon is centred and maximum at phase 0.2 , around the phase of the middle of the descending branch. It is the first time that such residual scatter of the radial velocity curve of RR Lyrae has been explicitly reported in a narrow phase interval. According to Chadid \& Gillet (1996a,b) the photosphere of RR Lyrea itself is crossed by a strong shock wave (main shock) during the phase interval of the descending branch of the heliocentric radial velocity curve. The shock intensity is at its maximum at the phase of the middle of the descending branch. This temporal physical phenomenon is a consequence of line broadening and doubling, which introduce a large deviation in the radial velocity measurements. This effect is particularly important when the shock intensity is maximum. The residual scatter phenomenon is consistent with previous results of Chadid (1997), which pointed out that important irregularities in the atmosphere of RR Lyrae occur during successive pulsation cycles, showing clearly significant differences, both in radial velocity and in line FWHM curves, from one pulsation cycle to the next. One of Chadid's plausible explanations of the irregularities is the nonlinear dynamical interaction between the motion of the highest and lowest metallic layers, when strong outward shock waves traverse them.

The largest dispersion is observed in the most abrupt phase of the radial velocity curve. To verify that the residual scatter is not a consequence of the frequency analysis method employed (caused by a bad fit of the descending branch of the curve), we performed a test by making an artificial signal of a general $\mathrm{RR} a b$ radial velocity curve, composed according to the following formula:

$$
\begin{aligned}
I(t)= & A_{0}+\sum_{i=1,15} A_{i} \sin \left[2 \pi \mathrm{i} f_{p} t+\Phi_{i}\right] \\
& +\sum_{i=1,7} B_{i} \sin \left[2 \pi\left(\mathrm{i} f_{p}+f_{B}\right) t+\Phi_{i}\right] \\
& +\sum_{i=1,7} C_{i} \sin \left[2 \pi\left(\mathrm{i} f_{p}-f_{B}\right) t+\Phi_{i}\right],
\end{aligned}
$$

to which we added white noise. We tested several frequency analyses assuming different levels of the noise and that there are some missing and wrong frequencies. These tests showed 
Table 4. Amplitude ratios of the harmonic components of the dominant radial pulsation mode, $H_{0}=A_{k f_{0}} / A_{f_{0}}$. Amplitude ratios of the modulation components, $H_{r}=A_{k f_{0}+f_{m}} / A_{f_{0}+f_{m}}$ and $H_{l}=A_{k f_{0}-f_{m}} / A_{f_{0}-f_{m}}$. Side lobe amplitude ratios, $S=A_{k f_{0}+f_{m}} / A_{k f_{0}-f_{m}}$. Degree of asymmetry and their corresponding uncertainties.

\begin{tabular}{ccclcc}
\hline \hline $\mathrm{k}$ & $H_{0}$ & $H_{r} \pm \sigma_{\mathrm{H}_{\mathrm{r}}}$ & $H_{l} \pm \sigma_{\mathrm{H}_{\mathrm{l}}}$ & $S \pm \sigma_{\mathrm{S}}$ & $Q \pm \sigma_{\mathrm{Q}}$ \\
\hline 1 & 1 & 1 & 1 & $5.2649 \pm 0.2376$ & $0.5430 \pm 0.0130$ \\
2 & $0.3810 \pm 0.0008$ & $0.4057 \pm 0.0074$ & $1.4706 \pm 0.0315$ & $1.4524 \pm 0.1924$ & $0.1845 \pm 0.0151$ \\
3 & $0.2478 \pm 0.0010$ & $0.3341 \pm 0.0083$ & $1.2558 \pm 0.0382$ & $1.4007 \pm 0.1192$ & $0.1669 \pm 0.0070$ \\
4 & $0.1691 \pm 0.0011$ & $0.3071 \pm 0.0118$ & $1.2074 \pm 0.0575$ & $1.3392 \pm 0.0508$ & $0.1450 \pm 0.0080$ \\
5 & $0.1108 \pm 0.0013$ & $0.2379 \pm 0.0158$ & $0.9471 \pm 0.0921$ & $1.3226 \pm 0.1003$ & $0.1388 \pm 0.0121$ \\
6 & $0.0719 \pm 0.009$ & $0.1831 \pm 0.0133$ & $0.6511 \pm 0.0399$ & $1.4806 \pm 0.0893$ & $0.1934 \pm 0.0060$ \\
7 & $0.0472 \pm 0.0012$ & $0.1447 \pm 0.0169$ & $0.5118 \pm 0.0330$ & $1.4883 \pm 0.0857$ & $0.1962 \pm 0.0071$ \\
8 & $0.0355 \pm 0.0010$ & $0.0972 \pm 0.0112$ & $\ldots$ & $\ldots$ & $\ldots$ \\
9 & $0.0310 \pm 0.0009$ & $0.0826 \pm 0.0015$ & $\ldots$ & $\ldots$ & $\ldots$ \\
10 & $0.0252 \pm 0.0012$ & $\ldots$ & $\ldots$ & $\ldots$ & $\ldots$ \\
11 & $0.0198 \pm 0.0011$ & $\ldots$ & $\ldots$ & $\ldots$ & $\ldots$ \\
12 & $0.0175 \pm 0.0008$ & $\ldots$ & $\ldots$ & $\ldots$ & $\ldots$ \\
13 & $0.0136 \pm 0.0008$ & $\ldots$ & $\ldots$ & $\ldots$ & $\ldots$ \\
14 & $0.0125 \pm 0.0010$ & $\ldots$ & $\ldots$ & & $\ldots$ \\
\hline
\end{tabular}

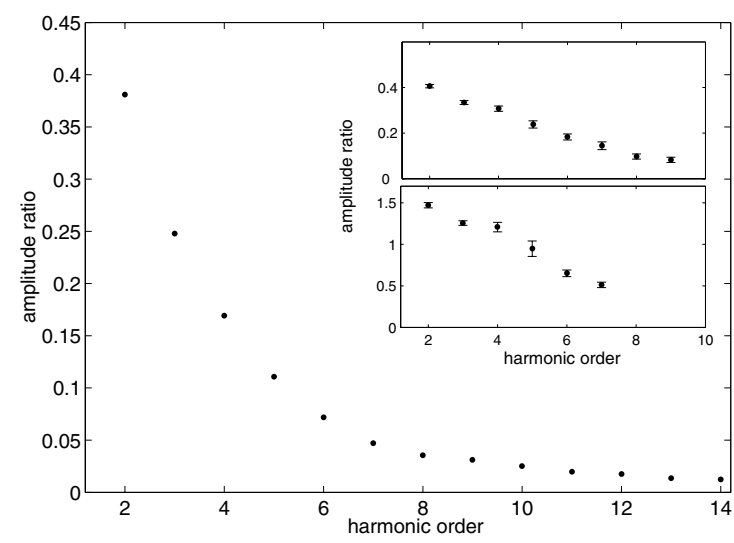

Fig. 5. Amplitude ratios of the harmonic components of the radial pulsation $A_{k f_{0}} / A_{f_{0}}$ compared to the amplitude ratios of the modulation components $A_{k f_{0}+f_{m}} / A_{f_{0}+f_{m}}$ (the upper insert panel) and $A_{k f_{0}-f_{m}} / A_{f_{0}-f_{m}}$ (the lower insert panel). The error bars are indicated, except for the errors of the amplitude ratios of the radial pulsation, which are smaller than the symbols.

that such errors in the analysis introduce only a very low dispersion during the descending branch of the radial velocity curve. Figure 10 shows this case with seven wrong frequencies.

A similar residual plot was shown by Lee \& Schmidt (2001) from photometric data of the Blazhko star DR Andromedea during the ascending branch of the light curve. More recently, Jurcsik et al. (2004) showed, using an extended CCD monitoring of the Blazhko star RR Gem, that the modulation of the light curves is concentrated in a very small phase interval - only $20 \%$ of the pulsation cycle - centred on the phase where $\mathrm{H}$ emission takes place, during the rise of the light curve.

\section{Discussion and conclusion}

The 2179 new high precision spectroscopic observation sets, spread continuously over a whole Blazhko cycle (41 days), give new spectroscopic results concerning the properties of the modulation of RR Lyrae. They show:

1. a triplet structure with an equidistant frequency spacing and a large asymmetry in the modulation amplitudes,

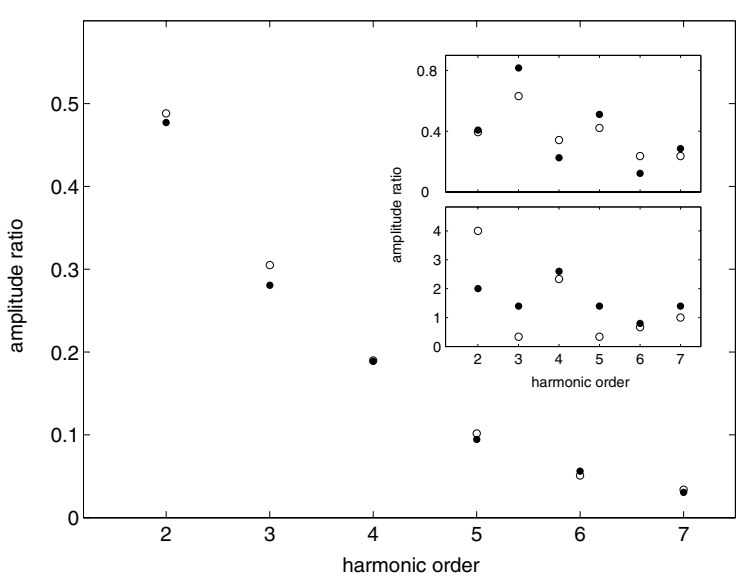

Fig. 6. Like Fig. 5 but from $B$ (solid circle) and $V$ (open circle) photometric data (Smith et al. 2003).

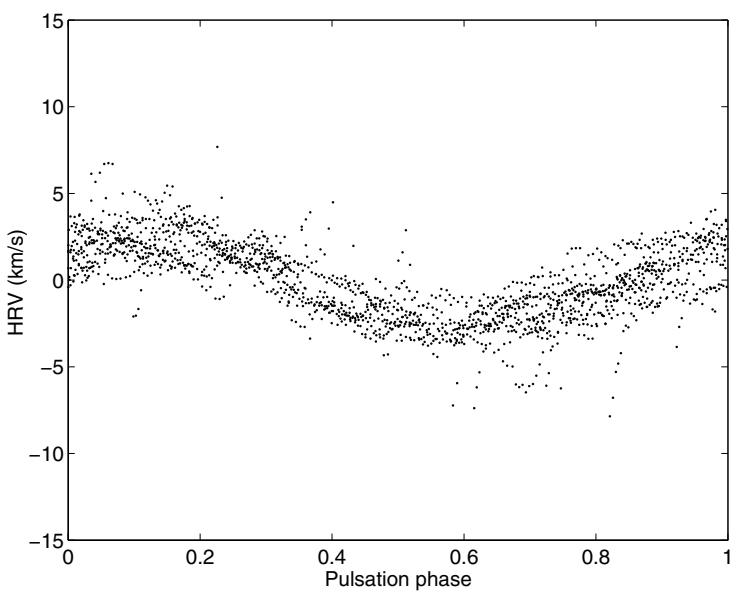

Fig. 7. Residual radial velocity curve after substraction of the main frequency $f_{0}$ and its harmonics. The pulsation phase has been calculated using $P=\left(f_{0}+f_{m}\right)^{-1}$ and $T_{0}=2451723.3769$.

2. the main pulsation amplitudes of the harmonic components decreasing exponentially, whereas the decrease is linear for the right and left modulation components,

3. no quintuplet structure detected within a median $1 \sigma$ radial velocity uncertainty of $250 \mathrm{~m} / \mathrm{s}$, 


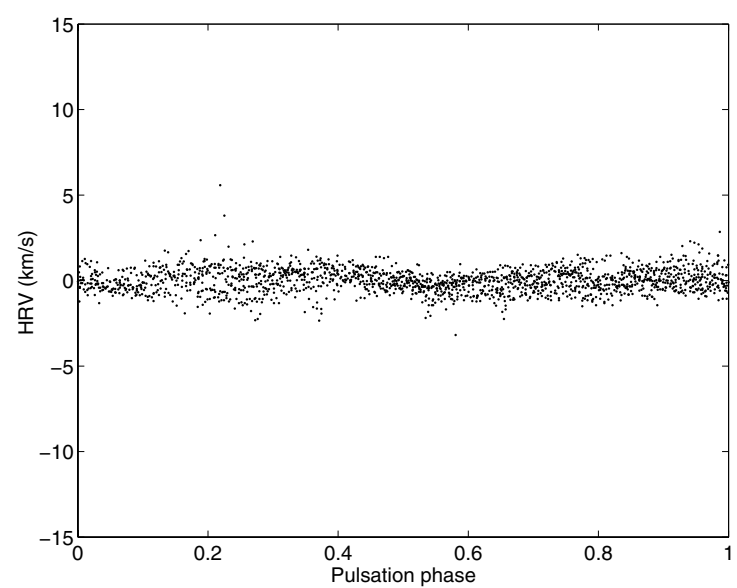

Fig. 8. Residual radial velocity curve after prewhitening with the radial pulsation and modulation frequencies $k f_{0}+f_{m}$. The pulsation phase has been calculated using $\left(f_{0}-f_{m}\right)^{-1}$ and $T_{0}=2451723.3769$.

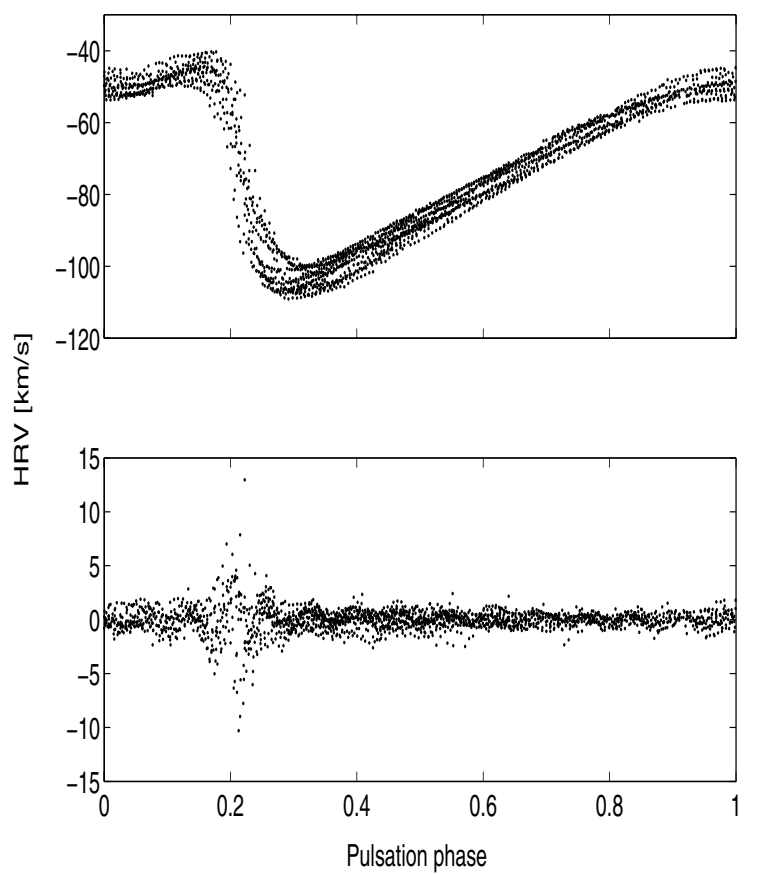

Fig. 9. The upper panel shows the heliocentric radial velocity curves from all 2179 spectra, folded with the pulsation phase calculated using the main frequency $f_{0}$ and $T_{0}=2451723.3769$. The lower panel is the residual radial velocity curve after prewhitening with the pulsation and modulation frequencies listed in Table 2 .

4. a residual scatter that occurs during a small phase interval corresponding to the phase of the main shock passage across the photosphere. The scatter is more important during the middle of the descending branch of the radial velocity curve, when the shock intensity is maximum, and

5. the radial velocity curves exhibit a Blazhko periodic variation in both heights of maxima and minima, in contradiction with photometric results, which indicate a Blazhko variation only in maxima of the light curves (see Preston et al. 1965; Smith et al. 2003).

The observational properties discussed above put constraints on any proposed explanation of the Blazhko enigma. The most

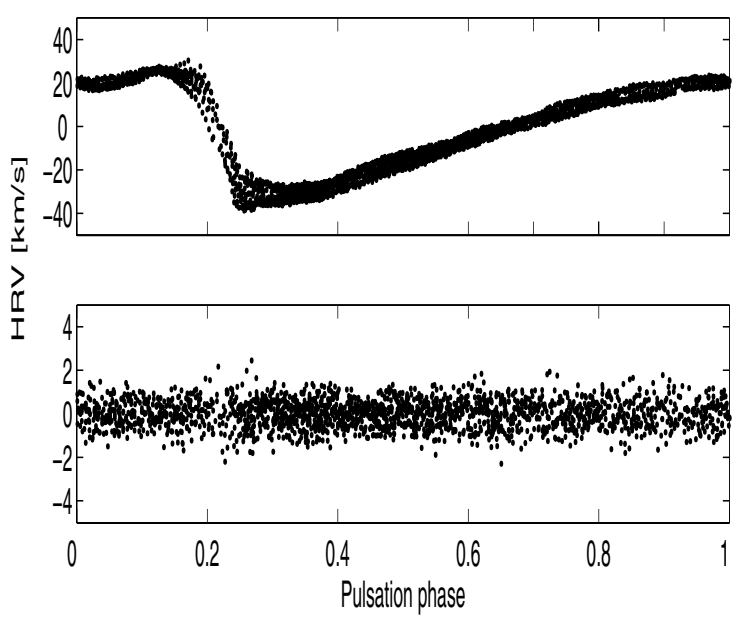

Fig. 10. Like Fig. 9, with an artificial signal.

popular hypotheses nowadays for explaining the observed properties of the Blazhko stars are focused on two groups:

(1) magnetic models, like the oblique pulsator model for roAp stars (Kurtz 1982). The magnetic models require a strong magnetic field and predict a quintuplet structure in the frequency spectrum (Cousens 1983; Shibahashi 2000; Shibahashi \& Takata 1995).

Recently, based on high-precision observations of the longitudinal magnetic field, Chadid et al. (2004) concluded that RR Lyrae is in fact a bona fide non-magnetic star, providing the strongest argument yet against the oblique magnetic rotator models. These models require surface field strengths of the order of $1 \mathrm{kG}$, a requirement inconsistent with the observational data.

Furthermore, our analysis does not show any quintuplet structures in the frequency spectrum below the $250 \mathrm{~m} / \mathrm{s}$ level. This is another argument against the magnetic models, which predict such structures.

(2) resonance models based on a nonlinear resonant coupling between the dominant radial mode and non-radial modes. The dipole modes have the highest probability of being nonlinearly excited (Nowakowski \& Dziembowski 2001; Nowakowski 2002; and Dziembowski; Mizerski 2005). These models predict a triplet frequency structure with sidelobes of similar amplitudes, symetrically placed around the fundamental radial mode frequency and its harmonics.

Neverthless, the results presented above also seem to rule out the resonance models, which are the only models still available to explain Blazhko phenomenon. In fact:

- One major problem for the resonance models is the production of strongly asymmetric modulation components detected in our data, since they predict approximate equality of the amplitude ratios between the symmetrically spaced side lobe frequencies of the different order components (Kovàcs 1995; Nowakowski \& Dziembowski 2001; Nowakowski 2002).

- It is important to recall that according to the nonlinear coupling theory (Nowakowski \& Dziembowski 2003), the nonradial modes are highly nonlinear in evolved stars. Thus, it is probably not easy for the resonance models, which involve nonradial pulsational modes, to explain why the high order nonlinear coupling terms show amplitudes commensurable with the first order types in our data (see Fig. 5).

- We can clearly see, from Fig. 5, that there are significant differences between the order dependence of the amplitudes of 
the modulation component frequencies and the radial mode frequency. The origin of these differences cannot be explained by nonlinear coupling. In fact, since the harmonic terms of the radial mode, invoked by nonlinear coupling, decrease nonlinearly, it is expected that the nonlinear coupling terms of nonradial modes have to decrease nonlinearly as well. Then, the nonlinear coupling can not explain the linear decrease of the amplitude modulation components.

- There are irregular changes in the RR Lyrae atmosphere during the Blazhko cycle. The residual scatter is most intense where the nonlinear effects are most important (shock wave passages). Therefore, the connection between temporal hydrodynamic phenomena and such modulation has to be taken into account in Blazhko models.

We conclude that the physical origin of the Blazhko phenomenon is still poorly understood. Our detailed spectroscopic observations of RR Lyrae, obtained over an entire Blazhko period, raise major complications for theoretical explanations of the Blazhko enigma, and provide new insight for understanding this long-standing puzzle. The connection of the atmospheric dynamics of RR Lyrae and the cycle modulations has to be seriously considered in future Blazhko theoretical investigations.

Acknowledgements. This work has taken advantage of the SIMBAD database, operated at CDS Strasbourg, France. We would like to express our gratitude to S. Jankov, D. Kurtz, J. Vernin \& G. Wade for reading of the manuscript.

\section{References}

Alcock, C., et al. 2003, ApJ, 598, 597

Blazhko, S. 1907, Astr. Nachr., 175, 325

Chadid, M. 2000, A\&A, 359, 991
Chadid, M. \& Gillet, D. 1996a, A\&A, 308, 481

Chadid, M., \& Gillet, D. 1996b, A\&A, 315, 475

Chadid, M., \& Gillet, D. 1997, A\&A, 319, 154

Chadid, M., Kolenberg, K., Aerts, C., \& Gillet, D. 1999, A\&A, 352, 201

Chadid, M., Wade, G. A., Shorlin, S. L. S., \& Landstreet, J. D. 2004, A\&A, 413, 1087

Cousens, A. 1983, MNRAS, 203, 1171

Dziembowski, W. A., \& Mizerski, T. 2005, Acta Astron., 54, 363

Deeming, T. J. 1975, ApSS, 36, 137

Jurcsik, J., Sodor, A., Varadi, M., Szeidl, B., et al. 2005, A\&A, 430, 1049

Kovacs, G. 1995, A\&A, 295, 693

Kovacs, G. 2001, Stellar pulsation-nonlinear studies, ed. M. Takeuti, \& D. D. Sasselov, Astrophysics and Space Science library (Dordrecht: Kluwer Academic Publishers), 257, 61

Kurtz, D. W. 1982, MNRAS, 200, 807

Lee, K. M., \& Schmidt, E. G. 2001, PASP, 113, 1140

Lenz, P., \& Breger, M. 2005, Co. Ast., 146, 53

Lucy, L. B., \& Sweeney, M. A. 1971, AJ, 76, 544

Moskalik, P., \& Poretti, E. 2003, A\&A, 398, 213

Nowakowski, R. M. 2002, in radial and Nonradial Pulsations as Probes of Stellar Physics, ed. C. Aerts, T. R. Bedding, \& J. Christensen Dalsgaard (ASP Conf. Ser., 259, San Francisco: ASP), IAU Coll., 185, 408

Nowakowski, R. M., \& Dziembowski, W. A. 2001, Acta Astron., 51, 5

Nowakowski, R. M., \& Dziembowski, W. A. 2003, Ap\&SS, 284, 273

Perryman, M. A. C. 1997, A\&A, 323, 49

Preston, G. W. 1967, The Magnetic and Related Stars, ed. R. C. Cameron, Mono Book Corporation, Baltimore, 26

Preston, G. W., Smak, J., \& Paczynski, B. 1965, ApJS, 12, 99

Shibahashi, H. 1994, What's new in the theory of stellar nonradial oscillations, ASP Conf. Ser., 76, 618

Shibahashi, H., \& Takata, M. 1995, ASP Conf. Ser., 83, 42

Shibahashi, H. 2000, The oblique pulsator model for the Blazhko effect in RR Lyrae stars, Theory of amplitude modulation I, ASP Conf. Ser., 203, 299

Smith, H. A., Matthews, J. M., Lee, K. M., et al. 1994, AJ, 107, 679

Smith, H. A., Barnett, M., Silbermann, N. A., \& Gay, P. 1999, AJ, 118, 572

Smith, H. A., Church, J. A., Fournier, J., et al. 2003, PASP, 115, 43

Szeidl, B., \& Kolláth, Z. 2000, The Impact of Large-Scale Surveys on Pulsating Star Research, ASP Conf. Ser., 203, 281 